\title{
New and evolving concepts of melanocytic nevi and melanocytomas
}

\author{
Iwei Yeh $\mathbb{D}^{1}$
}

Received: 19 July 2019 / Revised: 8 October 2019 / Accepted: 8 October 2019 / Published online: 28 October 2019

(c) The Author(s), under exclusive licence to United States \& Canadian Academy of Pathology 2019

\begin{abstract}
In daily clinical practice melanocytic nevi are commonly encountered. Traditionally, both benign and malignant melanocytic tumors have been sub-classified by their histopathologic characteristics with differing criteria for malignancy applied to each group. Recently, many of the mutations that initiate nevus formation have been identified and specific sets of mutations are found in different subtypes of nevi. Whereas a single mutation appears sufficient to initiate a nevus, but is not enough to result in melanoma, specific combinations of mutations have been identified in some melanocytic tumors that are regarded to be of low biologic potential. The term "melanocytoma" has recently been proposed by the World Health Organization to describe those tumors that demonstrate genetic progression beyond the single mutations that are found in nevi but are not frankly malignant. Melanocytomas occupy intermediate genetic stages between nevus and melanoma and likely have an increased risk of malignant transformation as compared to nevi. This review provides an update on the broad spectrum of melanocytic nevi and melanocytomas and outlines their key histopathologic and genetic features.
\end{abstract}

\section{Introduction}

This review will provide an update on the classification of melanocytic nevi based on recent genetic discoveries, as well as introduce the new concept of "melanocytomas" as melanocytic tumors that are intermediate between nevi and melanoma in terms of genetic progression. It is hoped that this review will assist pathologists in correctly identifying subcategories of melanocytic nevi, understanding the new concept of "melanocytomas" and choosing ancillary tests when the categorization or classification is uncertain, ultimately improving diagnosis and stratification of tumors for further treatment.

\section{Nevi}

Nevi are benign melanocytic tumors that can be broadly divided into those that arise in utero (congenital) and those that are acquired after birth (acquired). A single oncogenic

Iwei Yeh

iwei.yeh@ucsf.edu

1 Departments of Dermatology and Pathology, University of California, San Francisco, 1701 Divisadero St. Ste. 280, San Francisco, CA 94143, USA mutation within a melanocyte or melanocyte precursor initiates nevus formation with distinct initiating mutations corresponding to distinct histopathologic subtypes [1-5]. While this single oncogenic mutation leads to a period of clonal expansion, ultimately growth is inhibited by intact cellular safeguards. These cellular safeguards are disrupted by additional mutations in oncogenes and tumor suppressors during malignant progression [6]. The initiating oncogenic mutations define multiple distinct progression series from benign (nevi), intermediate (melanocytomas) to malignant (melanoma). We will first discuss acquired nevi followed by their congenital counterparts.

\section{Acquired nevi}

\section{Common (conventional) nevi}

Conventional or common nevi appear on the skin and uncommonly on the mucosa as small brown macules that may become raised over time. During their lifetime, an individual may develop tens to more than a hundred nevi. Typically appearing first in childhood, the number of nevi increases through the first three decades of life, and then decreases with age. There are genetic and environmental risk factors that lead to higher nevus counts and size. Genotypes associated with higher nevus count and size include those associated with decreased skin pigmentation, 
long telomeres, and cancer susceptibility (i.e. mutations in CDKN2A) [7, 8]. UV exposure, particularly intermittent UV exposure in childhood is associated with increased nevus counts $[9,10]$. Higher nevus counts are associated with an increased risk of death from melanoma [11].

Microscopically, common nevi contain small round melanocytes with uniformly staining nuclei and scant cytoplasm that may be entirely contained within the epidermis (junctional), located both within the epidermis and dermis (compound) or completely within the dermis (intradermal). Within the epidermis, melanocytes may be predominantly arrayed as nests or single cells (lentiginous pattern). Within the dermis, melanocytes may be limited to the superficial dermis or extend into the reticular dermis often surrounding adnexal structures. The latter distribution is referred to as the congenital growth pattern due to similarities with the distribution of melanocytes in congenital nevi. A key microscopic feature of common nevi is the presence of so-called "maturation" that refers to a decrease in melanocyte size, pigmentation, and the size of melanocyte aggregates with depth in the dermis. Common nevi at distinct anatomic sites may more commonly display microscopic features that can be worrisome for melanoma [12]. Nevi on the breast and flexures may have large and confluent junctional nests, limited pagetoid scatter, and superficial dermal fibrosis with a sparse inflammatory infiltrate $[13,14]$. Nevi on the scalp, particularly in adolescents, may have large discohesive junctional nests that are irregularly distributed and extend into follicular epithelium. Limited pagetoid scatter and superficial dermal fibrosis may also be present [15]. Genital nevi may have a markedly irregular growth pattern within the epidermis, occasionally with marked confluence of atypical and hyperchromatic melanocytes and similar features may be seen in the superficial dermal component [16]. Some acral nevi demonstrate a lentiginous growth pattern with significant pagetoid scatter limited to their central portions (Fig. 1) [17, 18].

Common nevi typically harbor $B R A F$ V600E mutations ( $\sim 5 \%$ ) with NRAS mutations present in a minority of cases [1]. The BRAF mutation affects one of two alleles in every melanocyte of the nevus, indicating that a nevus is the result of a clonal outgrowth of a single melanocyte that acquired the BRAF V600E mutation [3]. This concept can be confirmed by immunostaining with the VE1 antibody, specific for BRAF V600E that reveals the presence of this abnormality in each melanocyte of a $B R A F$-mutated nevus.

\section{Spitz nevi}

Spitz nevi appear as pink to red-brown, dome-shaped papules with sharply delimited edges, that have a predilection for the lower extremities [19]. These arise at an earlier age as compared to common nevi occurring most frequently in the first decade of life and then decrease in incidence with age [20-22]. In contrast to common nevi that typically display a slow evolution, Spitz nevi commonly have a short period of rapid growth over months before becoming stable in size [19]. The clinical differential diagnosis often includes verruca vulgaris or hemangioma.
Fig. 1 Common acquired nevi at "special" anatomic sites. a Scalp nevus with large nests of melanocytes with pale cytoplasm in the epidermis above papillary dermal fibrosis. b Genital nevus with large irregularly shaped nests within superficial dermal fibrosis. c Acral nevus with scatter of single cytologically bland melanocytes into the spinous layer of the epidermis. Flecks of pigment are arrayed within a column above the crista limitans
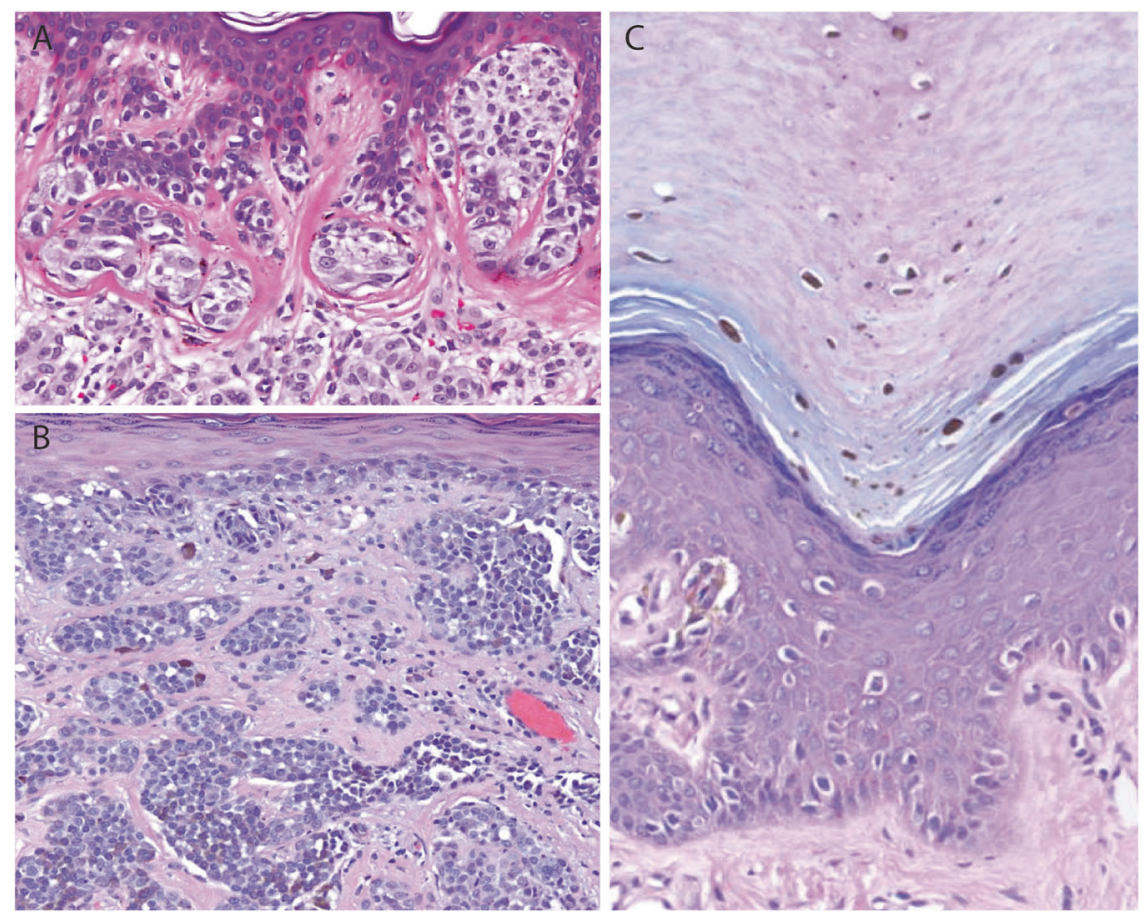
Basal cell carcinoma may be a clinical consideration in older patients. Genetic and environmental risk factors for Spitz nevus development have not been identified.

The presence of large spindled and/or epithelioid melanocytes is the defining histopathologic feature of Spitz nevi. When the epidermis is involved, Spitz nevi often show epidermal thickening with elongated rete ridges and hyperkeratosis. Epidermal nests of melanocytes may display clefts between the melanocytes themselves and from surrounding keratinocytes. The epidermis may contain Kamino bodies seen in hematoxylin and eosin stained sections as round to scalloped dull pink homogeneous aggregates. Almost half of Spitz nevi with an intraepidermal component contain Kamino bodies [19]. Importantly, Kamino bodies are not specific to Spitz nevi since they can also be seen in other types of nevi as well as melanoma. The scatter of melanocytes into the upper levels of the epidermis is a finding usually associated with the progression of nevi to melanoma but is not uncommon in Spitz nevi. First described as "juvenile melanoma" by Sophie Spitz in 1948 [23], it is now established that Spitz tumors fall in a spectrum from benign to malignant, with many tumors previously included in the original series of "juvenile melanoma" reclassified as Spitz nevi.

The mutations identified to date in Spitz nevi are distinct and more diverse than those in common nevi. They include activating mutations of HRAS and rearrangements of receptor tyrosine kinases (ROS1, ALK, MET, NTRK1, NTRK3, RET, MERTK) or serine/threonine kinases involved in the MAPK pathway (BRAF, RAF1, MAP3K8) that lead to constitutive kinase activity [5, 24-30]. Spitz nevi with HRAS mutations typically gain an extra copy of chromosome $11 \mathrm{p}$, resulting in an additional copy of the mutant HRAS allele. The copy number changes observed in other genetic subtypes of Spitz nevi are more variable as the kinase fusions that arise in Spitz nevi can involve a range of fusion partners and appear to arise as a consequence of different genetic mechanisms. When the kinase fusion is the result of a balanced translocation or inversion, copy number changes are not introduced as a byproduct. However, when the structural rearrangement is the result of an unbalanced translocation, deletion, tandem duplication, or even chromothripsis, copy number changes are introduced involving the chromosomes on which the rearranged genes reside. In our experience, copy number changes involving genomic regions near rearranged genes are not necessarily a sign of additional genetic progression and may be compatible with a diagnosis of Spitz nevus.

The expression of oncogenic kinase fusion genes is regulated by the promoter of the $5^{\prime}$ fusion partner. Given that many of the kinase fusion genes have a wide array of $5^{\prime}$ partners with the kinase itself typically on the $3^{\prime}$ end of the fusion gene, the kinase fusion genes may be expressed at different levels. In some Spitz nevi, there is subsequent gain of the fusion gene, similar to what is common for mutant $H R A S$, which results in copy number changes. While this leads to increased expression of the fusion kinase, it may not necessarily reflect an increased risk of progression.

The distinct oncogenic outputs of the different initiating oncogenes in Spitz nevi likely explains the phenotypic differences between genetic subtypes of Spitz nevi. Spitz nevi with $H R A S$ mutations are typically limited to the dermis without involvement of the epidermis. They typically have a plaque like configuration with a broad flat profile (Fig. 2a). They are characterized by nests and cords of fusiform to polygonal melanocytes with abundant cytoplasm and vesicular nuclei that are interspersed between thickened collagen bundles (Fig. 2b). Common to all Spitz nevi, dermal mitotic figures are not unusual and can cause further concern for malignancy. Since they often lack a junctional component and do not show the epidermal hyperplasia and Kamino bodies characteristic of Spitz nevi, HRAS-mutated Spitz nevi may not be recognized and be mistaken for melanoma. In these cases, evaluation of the genomic copy number can be helpful as $H R A S$-mutant Spitz nevi typically demonstrate gain of the short arm of chromosome 11 but no other copy number aberrations (Fig. 2c).

The majority of Spitz nevi are initiated by structural rearrangements resulting in kinase fusions. In contrast to other tumors initiated by structural rearrangements (i.e. chronic myelogenous leukemia with recurrent $B C R-A B L$ translocations), the kinases that are rearranged are highly diverse. Not only are the kinase genes themselves diverse, but so are their range of fusion partners. This diversity likely accounts for the variable histopathologic features observed in Spitz tumors and contributes to their diagnostic difficulty. Nevertheless, when examining tumors that are initiated by fusions of a specific kinase, common histopathologic patterns begin to emerge. For example, $A L K$ fused Spitz tumors have large junctional nests and elongated radially oriented fascicular nests (Fig. 2d-f) [31-33]. NTRK1-fused tumors demonstrate filigree-like rete ridges and rosette-like structures [33, 34].

Some of the kinases that are rearranged in Spitz tumors are not expressed in normal melanocytes (i.e. ALK and ROS1). When these fusions are present, immunohistochemistry using antibodies directed toward the kinase domain demonstrates staining in all the melanocytes of the Spitz nevus, demonstrating that the Spitz nevus is a clonal proliferation of a melanocyte transformed by the fusion. Other kinases are expressed in melanocytes (i.e. NTRK1 and NTRK3) but the expression level of the fusion gene is often markedly higher than that of the native kinase, and strong high intensity staining for the kinase domain of these proteins is predictive of the presence of a gene fusion. 

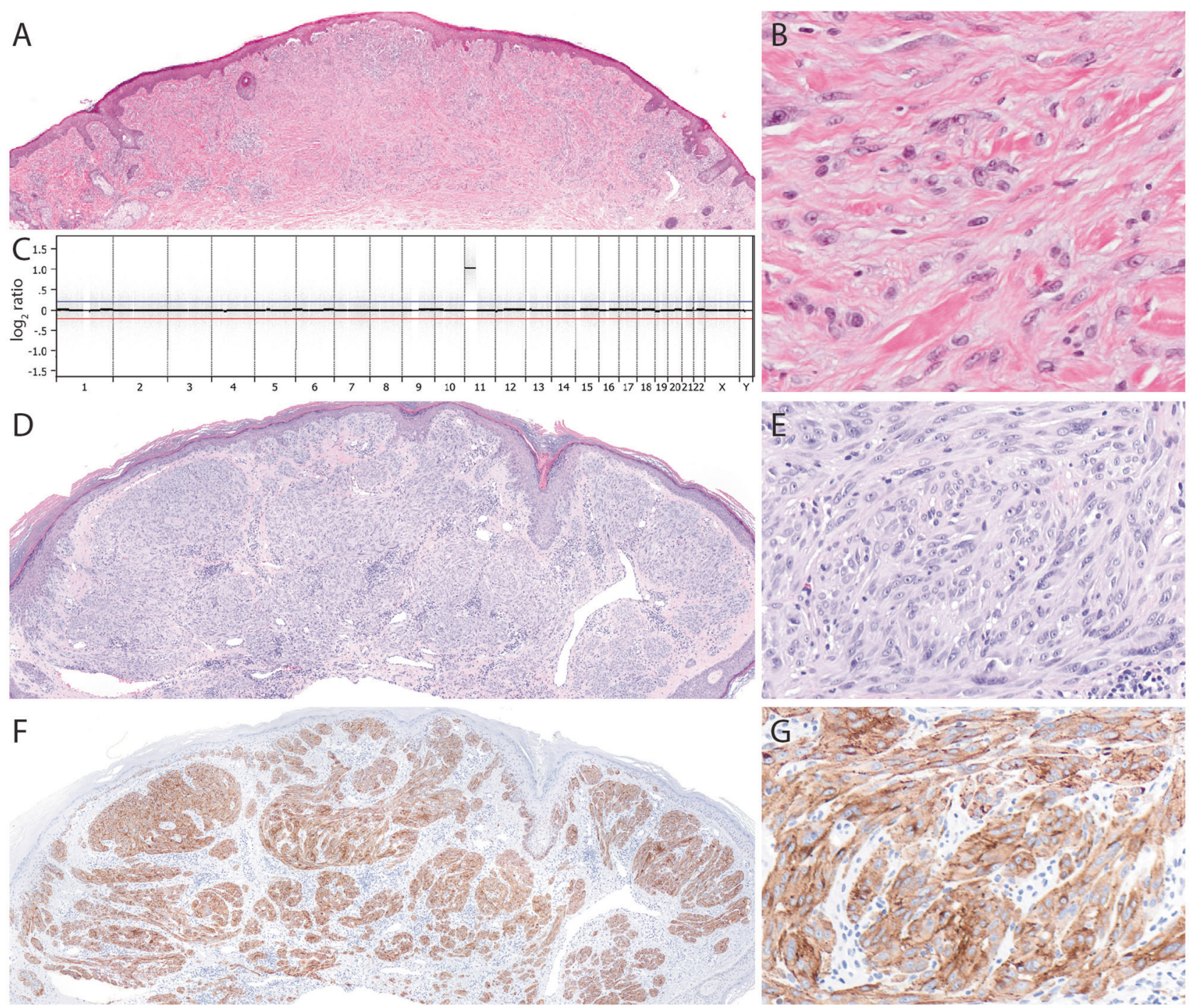

Fig. 2 Spitz nevi. a $H R A S$-mutant Spitz nevus with a broad flat-based profile. b Nests and cords of fusiform melanocytes are positioned between thickened collagen bundles within the dermis. $\mathbf{c}$ The genomic copy number profile demonstrates gain of chromosome $11 \mathrm{p}$, as is typical of $H R A S$-mutant Spitz nevi. d $A L K$-fused Spitz nevus with a domed surface and large nests in the superficial dermis. e On higher

\section{Blue nevi}

Blue nevi are named after their blue clinical appearance that is the result of pigmented dermal melanocytes and melanophages and the Tyndall effect. They typically occur as small round macules and papules on the face, scalp, and dorsal hands in an anatomic distribution that is distinct from common nevi. Blue nevi also occur internally, such as on the palate, cervix, and prostate. Genetic and environmental risk factors for blue nevus development have not been identified.

Common blue nevi are composed of sparsely distributed spindled pigmented melanocytes interspersed between magnification, the fascicular nests typical of $A L K$-fused Spitz nevus can be appreciated. $\mathbf{f}$ The melanocytes of the $A L K$-fused Spitz nevus display strong uniform expression of the kinase domain of ALK by immunohistochemistry. g On higher magnification, the fascicular configuration of the nests is highlighted

collagen bundles and elastic fibers; often, the collagen bundles demonstrate sclerosis (Fig. 3a, b) [12]. Sometimes the spindled melanocytes have a periadnexal distribution. Cellular blue nevi have a second component that consists of pale ovoid melanocytes arrayed in nests and fascicles (Fig. 3c, d). The melanocytes within blue nevi have a uniform appearance, regardless of their depth within the dermis and thus lack so-called "maturation". A significant number of melanophages are typically present within a blue nevus, although hypopigmented forms may occur.

Blue nevi harbor oncogenes that activate the $\mathrm{G}$ alpha $\mathrm{q}$ pathway. Activating mutations in GNAQ are the most common initiating mutation in blue nevi with activating 

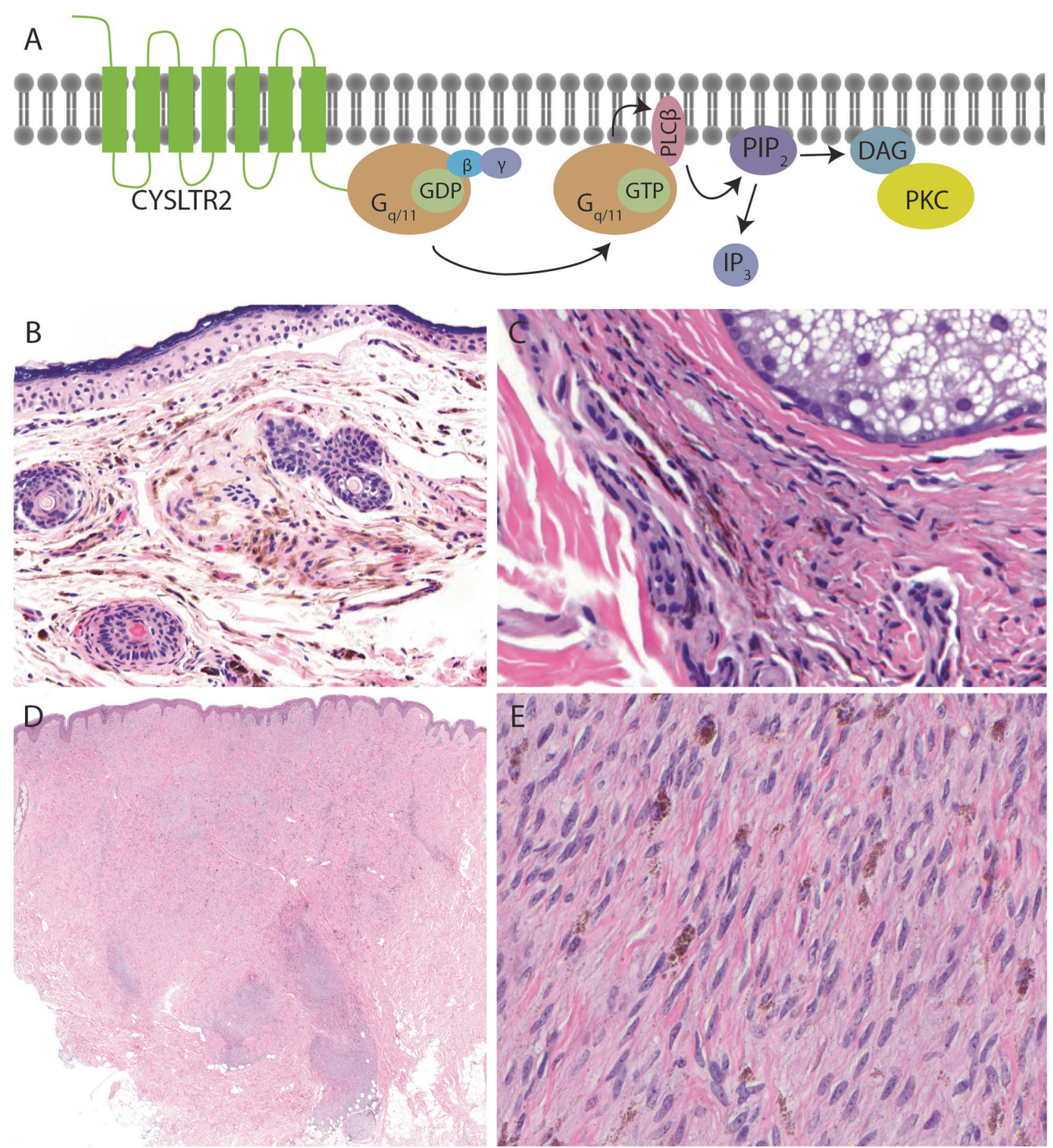

Fig. 3 Blue nevi. a Overview of the $\mathrm{G}$ alpha q signaling pathway. Gprotein coupled receptors (such as CYSLTR1) activate $\mathrm{G}_{\mathrm{q}}$ or $\mathrm{G}_{11}$ alpha subunits. The active GTP-bound alpha subunit activates phospholipase $\mathrm{C}$ beta which cleaves phosphatidylinositol 4,5-bisphosphate $\left(\mathrm{PIP}_{2}\right)$, yielding the second messengers inositol trisphosphate $\left(\mathrm{IP}_{3}\right)$ and diacylglycerol (DAG). DAG binds to and activates protein kinase $\mathrm{C}$

mutations in its homolog GNA11 present in a minority of blue nevi $[2,4,35]$. More recently, activating mutations in CYSLTR2 and PLCB4 which encode proteins that function upstream and downstream of GNAQ and GNA11 in the G
(PKC). b Blue nevus with sparsely distributed dendritic melanocytes in the dermis. c Dendritic melanocytes may have a periadnexal distribution. d Cellular blue nevus with more cellular areas in its lower portion. e Pale ovoid melanocytes arrayed in fascicles and nests are characteristic of cellular blue nevus

alpha Q pathway, respectively (Fig. 3e), have also been identified in a small subset of blue nevi $[35,36]$. These mutations occur in a mutually exclusive pattern with only one initiating mutation present in each nevus. 
Mutations that activate the $G$ alpha $q$ pathway in blue nevi are also found in melanocytic tumors of the uveal tract and leptomeninges. The uveal tract typically contains dendritic melanocytes that are not associated with epithelium. Thus, $\mathrm{G}$ alpha q pathway activating mutations may transform a distinct set of melanocytes that are not associated with epithelium [37].

\section{Dermal melanocytoses}

Dermal melanocytoses present on the skin or mucosal surfaces as light, evenly colored patches with shades of blue, green, gray, and brown. They may be confused with bruising. More common in Asians, most present at birth (discussed with congenital nevi below) but they may also arise in adulthood. Environmental risk factors for dermal melanocytosis development have not been identified.

Microscopically, dermal melanocytoses contain pigmented dendritic melanocytes within the dermis but more sparsely distributed than in blue nevi and with only rare melanophages [12]. Some dermal melanocytoses occur as a consequence of the acquisition of a GNAQ or GNA11 activating mutations within a melanocyte or melanocyte precursor [2, 4]. Thus, they have overlapping features with blue nevi but differ in their clinical presentation as large faintly pigmented patches and the sparser distribution of melanocytes within them.

In some cases, dermal melanocytoses involve tissues innervated by particular nerve distributions. For example, the nevus of Ota affects tissues innervated by the ophthalmic (V1) and maxillary (V2) distributions of the trigeminal nerve and the nevus of Ito affects tissues innervated by the acromioclavicular nerve. Other nerve distributions may also be affected. Nevus of Ota may involve the sclera, choroid, and mucosal membranes of the nose, mouth, and ear and is rarely associated with leptomeningeal melanocytosis [38]. Dermal melanocytoses that arise along nerve distributions suggest that they may arise due to somatic mutation in a nerve-derived melanocyte precursor. Therefore, what distinguishes dermal melanocytoses from blue nevi may be the cell of origin. Occasionally, dermal melanocytoses may contain foci with increased melanocytes in a periadnexal distribution, resembling blue nevi. Further investigation into the cell of origin or the spectrum of initiating mutations in GNAQ, GNA11, CYSLTR2, and PLCB4 may help determine what distinguishes blue nevi from dermal melanocytosis.

\section{Congenital nevi}

Congenital nevi arise during development as a result of an in utero oncogenic initiating mutation within a melanocyte precursor. Typically present at birth, they may also present in infancy, presumably due to darkening or expansion of neoplastic melanocytes present at birth.

\section{Common congenital nevi}

sWe introduce here the term common congenital nevus to specifically refer to congenital nevi that contain NRAS or $B R A F$ hotspot mutations, since there are other distinct and less common types of congenital nevi without these aberrations (discussed below). Most common congenital nevi present as brown to black papules and plaques with variegated coloration, a rugose surface and hypertrichosis, characterized by long terminal hairs. Estimated to occur in $0.6-1.5 \%$ of the population [39], large or giant congenital nevi are defined as those that involve $>6 \%$ of the patient's total body surface area or exceed $20 \mathrm{~cm}$ in diameter [40, 41]. Approximately 1 in 20,000 newborns are affected by large or giant congenital nevi that can result in significant functional impairment and disfigurement. Smaller common congenital nevi often cannot be distinguished from common acquired nevi except by their clinical history. In a single study, black ethnicity was associated with an increased risk of congenital nevi [40]. Other genetic and environmental risk factors for common congenital nevus development have not been identified.

Microscopically, common congenital nevi are composed of small round melanocytes that resemble those that form a common nevus. They may be junctional or compound with a dermal component often extending into the deep dermis and into the deep subcutis or fascia. Dermal melanocytes often assume a periadnexal distribution. Most large and giant congenital nevi contain NRAS Q61 mutations ( 80\%) with a minority harboring the $B R A F$ V600E mutations that predominate in smaller congenital nevi [42, 43]. We have theorized that the initiation of a melanocyte earlier in its development leads to a larger congenital nevus, suggesting that melanocyte precursors at earlier stages of development may be more susceptible to transformation by acquisition of NRAS mutation than BRAF mutation.

Smaller "satellite" nevi can be numerous and widely distributed in the skin distant from large or giant congenital nevi. These satellite nevi harbor the same initiating mutation as the central nevus, indicating that they derive from the same transformed clone of melanocytes but separate from the main tumor during development. Congenital nevi are clinically staged based on their size, the presence of satellite nevi, involvement of other organ systems such as the CNS, and other clinical features [41]. This risk of melanoma development within a congenital nevus likely reflects the number of partially transformed melanocytes, each with a small likelihood of progressing to melanoma. Large and giant common congenital nevi carry a lifetime risk of melanoma that ranges from $2.5 \%$ to $8 \%$. 


\section{Congenital nevi with Spitz nevus mutations}

Nevus spilus or speckled lentiginous nevus presents at birth as a light tan patch. Papules develop within the tan patch over time that may become raised. HRAS mutations have been identified in nevus spilus, with copy gain of chromosome $11 \mathrm{p}$ harboring the mutant $H R A S$ allele present within the papules that develop within the lesion [44].

Rearrangements of the genes coding for the $B R A F$, $R A F 1$, and $A L K$ kinases have been reported in congenital nevi [45-47]. The kinase fusion genes are similar to those that occur in Spitz nevi, but the histopathologic features of congenital nevi with Spitz nevus initiating mutations and their natural history has yet to be determined.

\section{Congenital blue nevi}

Congenital blue nevi are rare, with only a few cases having been reported in the literature [48-51]. They are reported to present as deeply pigmented patches and plaques, commonly on the scalp. Their histology is similar to that of acquired blue nevi, and they often display areas of cellular blue nevus, characterized by fascicles of fusiform melanocytes. They may involve deep bony structures of the central nervous system [52-56]. Notably the GNAQ and GNA11 mutations that are typical of blue nevi are also found in primary melanocytic tumors that arise in the leptomeninges [57].

\section{Congenital dermal melanocytoses}

Congenital dermal melanocytoses commonly present over the lumbosacral area in a symmetric distribution. This pattern is more common in Asian, African, and Native American populations, and was previously referred to as "Mongolian spot" since at the time of its original description, it was thought to be limited to Asian populations. Congenital dermal melanocytosis over the lumbosacral area occurs in greater than two-thirds of newborns in some ethnic populations but typically resolves by puberty. These cases do not appear to reflect a neoplastic process that is initiated by somatic mutation, since it would be unlikely to occur in a large fraction of certain populations at a specific anatomic site. Likewise, the diffuse dermal melanocytosis that is associated with lysosomal storage diseases or mucopolysaccharidoses may not be a neoplastic condition.

However, some congenital dermal melanocytoses do not self-resolve and are more likely to represent neoplasms. Ectopic or aberrant congenital dermal melanocytoses, which occur at sites other than the lumbosacral area, are most common on the extremities, upper back or chest, may be particularly large or deeply pigmented and are less likely to resolve. Nevus of Ota and nevus of Ito may also present at birth and do not self-resolve.

\section{Congenital nevi with associated vascular proliferations}

Congenital nevi can be seen in combination with vascular proliferations in the condition termed phakomatosis pigmentovascularis [58, 59]. BRAF, NRAS, GNAQ, and GNA11 mutations can initiate congenital and acquired vascular neoplasms as well as melanocytic neoplasms [60-62]. At least in some cases, the same initiating mutation has been identified in both the melanocytic and vascular birthmark, suggesting that phakomatosis pigmentovascularis is a clonal process with two distinct lines of differentiation, perhaps arising due to a mutation in a multipotent stem cell.

\section{Melanocytomas}

Some tumors that were classified as nevi or melanocytic tumors of low or uncertain biologic potential are now known to harbor multiple pathogenic mutations. In addition to mutations that activate the MAPK pathway, a key signaling pathway in melanocytic neoplasia altered in nevi, they harbor additional mutations that affect other biological processes and lead to phenotypic changes. Because these tumors have multiple driver mutations, they have been reclassified as melanocytomas, either low-grade or highgrade in the most recent WHO Classification of Skin Tumors to indicate that they may carry a higher risk of malignant transformation [63]. As the risk of progression of melanocytomas is not well characterized, we will discuss them as a single group, keeping in mind that they will likely be later segregated into lower and higher risk categories. Many melanocytomas arise within a preexisting nevus, due to the acquisition of a secondary pathogenic mutation in a single melanocyte which leads to subclonal outgrowth and distinct histopathologic features resulting in a so-called "combined" nevus. Further studies are needed to determine their risk for transformation and optimal clinical management.

\section{Pigmented epithelioid melanocytomas (PRKAR1A- inactivated melanocytomas)}

Pigmented epithelioid melanocytoma was proposed as an entity in 2004 to describe low-grade melanocytic tumors that are composed of a mixture of epithelioid and spindled melanocytes and are heavily pigmented with many interspersed melanophages [64]. In its initial description, pigmented epithelioid melanocytoma included epithelioid blue nevus associated with Carney complex and animal-type melanoma. 
Carney complex is a genetic syndrome that is associated with an increased number of epithelioid blue nevi and is due to germline inactivating mutations in the alpha 1 regulatory subunit of protein kinase A (PRKARIA). PRKAR1A is expressed in other melanocytic tumors, but is lost in most pigmented epithelioid melanocytomas as demonstrated by immunohistochemistry [65]. The clinical course appears benign, despite subclinical deposits in the sentinel lymph nodes of almost a third of patients [66]. Recent genetic studies demonstrated that tumors classified as pigmented epithelioid melanocytoma by their histopathologic features are genetically diverse $[67,68]$. Notably, some harbored BRAF V600E mutations and bi-allelic inactivation of the tumor suppressor PRKARIA [69]. In some of the remaining tumors, only a single oncogenic alteration was identified (such as GNAQ, $M A P 2 K 1$ mutation, or PRKCA fusion) and these tumors may represent blue or other types of nevi.

Moving forward, we propose that the term PRKARIAinactivated melanocytoma, be used to refer specifically to those tumors with PRKARIA inactivation in addition to an initiating mutation, such as BRAF V600E. This term would replace the term epithelioid blue nevus to describe the melanocytic tumors associated with Carney complex. We restrict the use of the term blue nevus to refer to tumors that harbor mutations in the $\mathrm{G}$ alpha $\mathrm{Q}$ pathway; the tumors that arise in Carney complex typically do not harbor $\mathrm{G}$ alpha $\mathrm{Q}$ activating mutations but do demonstrate loss of the wildtype PRKARIA allele in combination with an initiating mutation such as BRAF V600E. Given their new genetic definition, further work to detail the features of PRKARIAinactivated melanocytoma will be needed to determine if these tumors have characteristic features that separate them from other deeply pigmented tumors.

\section{Deep penetrating melanocytomas (beta-catenin- activated melanocytomas)}

The deep penetrating nevus was first described by Helwig and colleagues at the Armed Forces Institute of Pathology (AFIP) in 1989 [70]. Almost a third of their series consisted of cases that had previously been diagnosed as melanoma. Clinically, these tumors appear as dark brown or blue papules, often distributed on the head, neck, and trunk. Deep penetrating nevi consist of loosely organized nests of melanocytes with vacuolated pigmented cytoplasm and vesicular nuclei that often descend deeply into the reticular dermis, sometimes with a bulbous lower border ("dumbbell shaped" pattern). There are typically many melanophages and lymphocytes within and around the nests of melanocytes. Notably, a decrease in melanocyte size and pigmentation and the size of melanocytic nests does not occur with increasing depth within the dermis and this lack of "maturation" can raise concern for melanoma. Tumors with components resembling deep penetrating nevus and common nevus are not uncommonly encountered in clinical practice and are often accompanied by a clinical history of a dark area appearing within a pre-existing nevus.

Due to the presence of many dermal melanophages and their lack of maturation, deep penetrating nevus was considered by some to be closely related to blue nevus. Others considered deep penetrating nevus more closely related to Spitz nevi as they are both composed of enlarged melanocytes with epithelioid cytomorphology. Deep penetrating nevi are not genetically related to blue nevi as they do not harbor activating mutations in the $\mathrm{G}$ alpha $\mathrm{Q}$ pathway. Instead, deep penetrating nevi have activating $B R A F, M A P 2 K 1$, or less commonly HRAS mutations that activate the MAPK pathway and thus in most cases are related to common acquired nevi. Deep penetrating nevi are characterized by concomitant genetic activation of the beta-catenin pathway, most commonly due to missense mutations in CTNNB1 [71]. An alternative genetic mechanism of beta-catenin activation is inactivation of the tumor suppressor $A P C$, which has been observed in a few cases of deep penetrating nevi that do not harbor $C T N N B 1$ mutation. Notably, the one reported case with activating CTNNBI and HRAS mutations demonstrated overlapping features of DPN and HRAS-mutant Spitz nevus. Thus, beta-catenin activation characterizes deep penetrating nevus, and due to the presence of more than a single pathogenic mutation these tumors have been tentatively reclassified as deep penetrating melanocytomas and typically belong to the common nevus progression series.

The genetics also explains so-called "combined nevi" with a deep penetrating melanocytoma component. These tumors have also been referred to as inverted type-A or clonal nevi and are often accompanied by a clinical history of a black area arising within a pre-existing nevus [72, 73]. When a single melanocyte of a common nevus acquires an activating CTNNB1 mutation, there is clonal expansion of that melanocyte with acquisition of the cellular phenotype of deep penetrating melanocytoma. However, the deep penetrating melanocytoma component may not extend into the deep dermis. Instead of using the term "combined" nevus to describe these lesions which does not allude to their genetic relationship, we currently refer to these lesions as deep penetrating nevus/melanocytoma, arising within a common acquired nevus. Perhaps in the future the field will adopt the term beta-catenin-activated melanocytoma, to better capture the genetic mechanism at work and recognize that many tumors with the same genetics and cytomorphology do not extend deeply into the dermis.

Deep penetrating melanocytomas demonstrate a distinct pattern of beta-catenin expression, whether they are observed in isolation or within a common acquired nevus (Fig. 4). By immunohistochemistry, the melanocytes of show high levels of cytoplasmic beta-catenin expression 


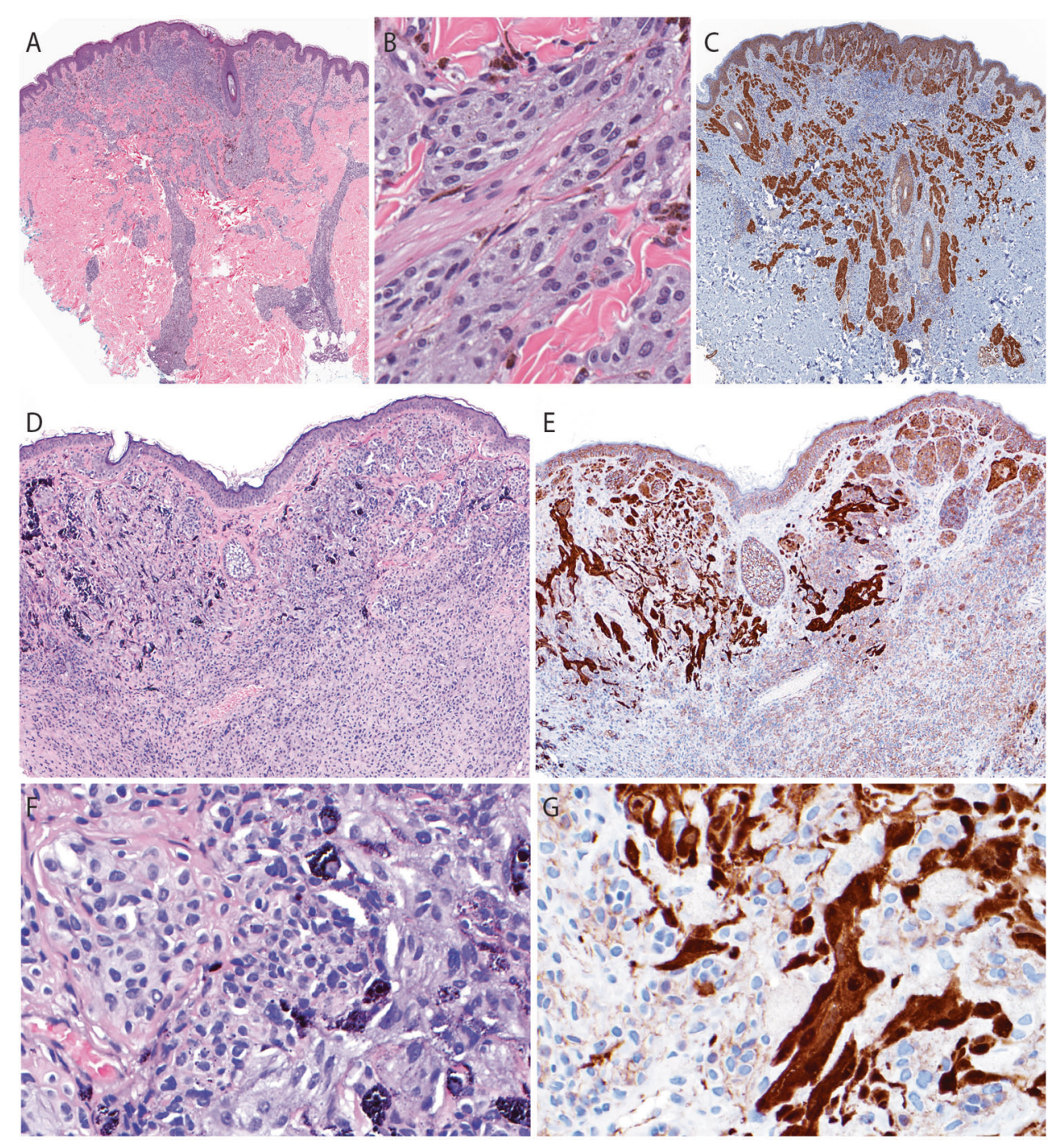

Fig. 4 Deep penetrating (beta-catenin activated) nevus/melanocytoma. a Low power view shows extension of the melanocytoma into the reticular dermis with melanocytes extending around adnexal structures. b Characteristic cytology is apparent at higher magnification. The melanocytes have moderate amounts of vacuolated and lightly pigmented cytoplasm and slight nuclear pleomorphism. They are arrayed in oval to fusiform nests with a somewhat plexiform pattern. There are scattered melanophages. c Beta-catenin immunohistochemistry demonstrates strong cytoplasmic (and nuclear) staining of the melanocytes. The staining is uniform throughout the melanocytoma. d A superficial focus of beta-catenin-activated melanocytoma arising

and occasionally strong nuclear beta-catenin expression. Notably, the levels of beta-catenin within melanocytes is uniform in deep penetrating nevus. By contrast, in common nevi, beta-catenin levels are increased in melanocytes near the epidermis or around adnexal epithelium.

While for the most part, deep penetrating melanocytomas have a benign biologic behavior, rare cases have been reported to result in metastasis and death even after consensus diagnosis by a panel of experts [71, 74]. Genetic analysis of two melanoma metastases from such cases within a congenital pattern common acquired nevus, low power view. e Beta-catenin immunohistochemistry shows intense cytoplasmic (and nuclear) staining of fusiform nests of melanocytes. In contrast, within the common acquired nevus the superficial melanocytes show patchy positivity and melanocytes deeper in the reticular dermis show little positivity. f High power view demonstrates two intermixed populations of melanocytes with those of the beta-catenin-activated melanocytoma showing features similar to those in $\mathbf{b}$ and adjacent small round melanocytes with scant, pale cytoplasm. $\mathbf{g}$ The two populations of melanocytes show distinct patterns of beta-catenin expression

demonstrated additional genetic alterations beyond the typical mutations that lead to MAPK and beta catenin pathway activation, indicating further transformation occurred [71]. Thus, genetic analysis may be helpful for further classifying unusual beta-catenin-activated tumors.

\section{BAP1-inactivated melanocytomas}

$B A P 1$-inactivated nevi/melanocytomas harbor bi-allelic inactivation of the tumor suppressor BAPI in combination 
with activating $B R A F$ or NRAS mutation [75-77]. Clinically, they appear as tan, pink or flesh colored dome-shaped papules, sometimes arising within a pre-existing brown flat nevus. The BAPl-inactivated melanocytoma is characterized by epithelioid melanocytes with glassy amphophilic cytoplasm and eccentrically placed nuclei. There is considerable variability in cell and nuclear size. The cellular plasma membranes appear distinct and there is often a permeative lymphocytic infiltrate (Fig. 5). In the majority of cases, dermal mitoses are present, sometimes even in the deeper portions of the tumor [78]. In most cases, BAPI inactivation occurs by a truncating mutation in $B A P l$ with loss of the wild-type allele. This may be due to loss of a portion of chromosome $3 p$, loss of the entire short arm of chromosome 3 , or loss of chromosome 3 . Immunohistochemistry for BAP1 typically demonstrates loss of nuclear BAP1 expression. However, this assay may not be fully sensitive for BAP1 inactivation because while BAPl is commonly inactivated by a truncating mutation, pathogenic missense mutations may occur instead, and these would be expected to result in expression of BAP1 protein with impaired function. Copy loss of BAPl involving part or all of chromosome 3 is typical and can be a clue to the diagnosis. Similar to deep penetrating (beta-catenin activated) melanocytomas, BAPI-inactivated melanocytomas may arise within a common acquired nevus.

In our experience, $B A P 1$-inactivated melanocytomas are encountered less frequently than deep penetrating (beta-catenin activated) melanocytomas, and this likely reflects both the concerning clinical appearance of deep penetrating (beta-catenin activated) melanocytomas prompting biopsy, as well as the fact that beta-catenin activated melanocytomas typically harbor a single additional activating mutation (CTNNB1 mutation) instead of the two additional alterations (mutation of $B A P 1$ followed by loss of the wild-type allele) in BAPl-inactivated melanocytomas and thus may be more prevalent. Germline pathogenic mutations in BAPl result in a cancer predisposition syndrome, with increased risks of cutaneous and uveal melanoma, renal cell carcinoma, mesothelioma, and other cancers [79-81]. Patients with a germlineinactivating mutation of $B A P 1$ may have multiple $B A P 1$ inactivated melanocytomas.

Prior to the identification of $B A P I$-inactivation in these tumors, they were thought to be in the Spitz category, and often designated as halo Spitz nevi [82] or atypical Spitz tumors [78, 83]. It is now clear that while they contain epithelioid melanocytes, they are not genetically related to Spitz nevi. The biologic potential of BAPl-inactivated melanocytomas appears low-grade, with most, in our experience, not recurring after biopsy. In contrast, tumors in the blue nevus category with inactivation of $B A P I$ are highly worrisome for blue nevus like melanoma.



Fig. $5 B A P 1$-inactivated nevus/melanocytoma. a $B A P 1$-inactivated melanocytoma arising within a common acquired nevus. Nests of small melanocytes are present in the superficial aspect (common acquired nevus). Larger melanocytes with abundant cytoplasm are present deeper within the dermis. b BAP1 immunohistochemistry demonstrates nuclear BAP1 expression in the melanocytes of the common acquired nevus, but not the $B A P 1$-inactivated melanocytoma. c $B A P 1$-inactivated melanocytomas are characterized by epithelioid melanocytes with pale pink cytoplasm, well-defined cellular membranes and nuclear pleomorphism. Some nuclei are eccentrically located within melanocytes

\section{Atypical spitz tumors (melanocytomas)}

Historically, the term atypical Spitz tumor has been used to describe tumors with spitzoid cytomorphology that were not clearly benign or malignant. These tumors have a high rate of dissemination to the draining lymph nodes (as determined by sentinel lymph node biopsy), but typically have 


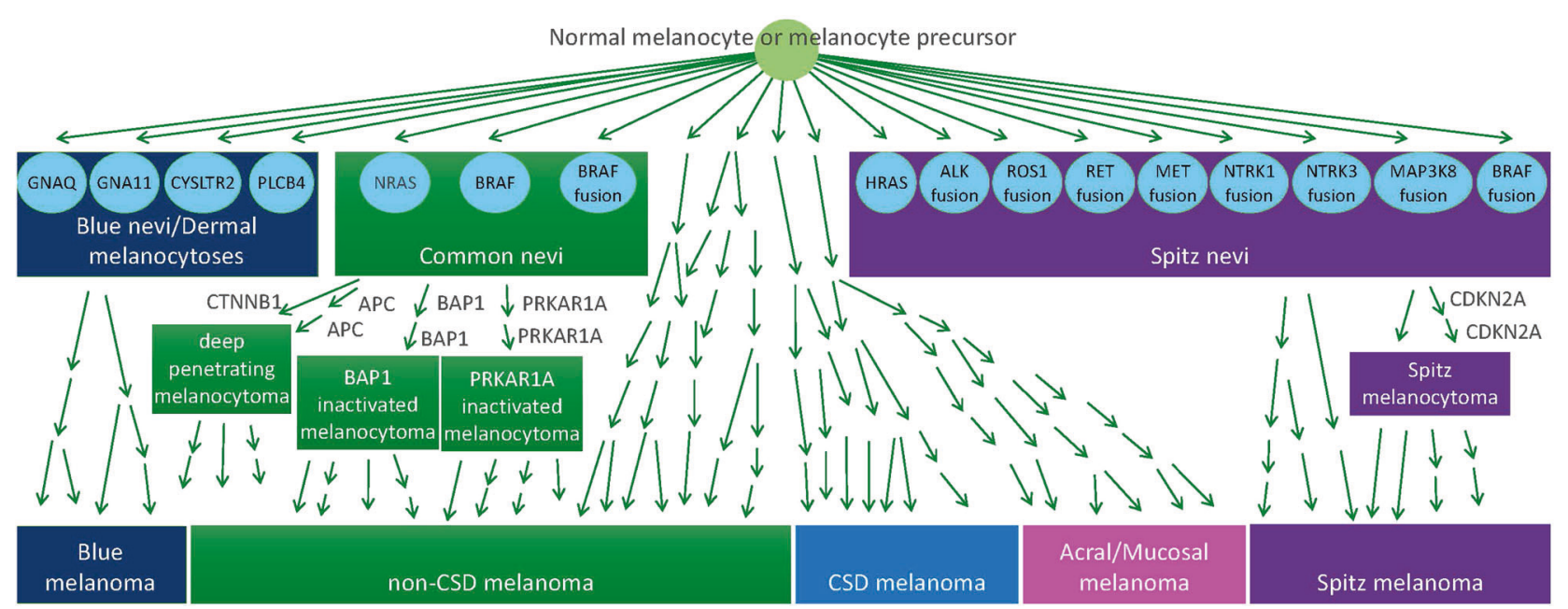

Fig. 6 Genetic classification of nevi, melanocytomas, and melanomas. A single initiating mutation is found in nevi, with different initiating mutations corresponding to different subtypes. Combinations of mutations occur in melanocytomas which often arise from pre-existing

benign biologic behavior without clinical recurrence $[84,85]$. In the most recent WHO classification of skin tumors, the definition of Spitz tumors has been revised to include only tumors that contain the initiating oncogenes of Spitz nevi. Thus, moving forward, atypical Spitz tumors will no longer include tumors with $B R A F$ or $N R A S$-activating mutations, which are present in a subset of atypical Spitz tumors as defined by cytomorphology [86-88].

Additional genetic alterations that have been identified in Spitz tumors (in addition to Spitz nevus initiating mutations) include homozygous deletion of CDKN2A, TERT promoter mutations, structural rearrangements in the regulatory regions of TERT and copy number losses and gains [89-91]. In an initial study, a Spitz tumor with $B R A F$ rearrangement and TERT promoter mutation was lethal [90] and additional studies are needed to further determine the prognostic significance of TERT promoter mutations and loss of $C D K N 2 A$ in Spitz tumors. In melanocytic tumors initiated by $B R A F$ or NRAS mutation, isolated CDKN2A loss or TERT promoter mutation can arise early in genetic progression, before full malignant transformation [92].

The management of atypical Spitz tumors has shifted from the routine use of sentinel lymph node biopsy which often resulted in upstaging to a diagnosis of melanoma and adjuvant therapy when tumor was identified in the sentinel node to conservative management with re-excision if feasible. Despite this shift, the diagnosis of atypical Spitz tumor still causes a great deal of consternation, particularly when made in a young child. It is likely that as we learn more about the histopathologic features and natural history of Spitz nevi initiated by different oncogenes, many Spitz tumors that are currently classified as atypical Spitz tumors may be recategorized as Spitz nevi. Additional studies are nevi. Melanomas on skin with high amounts of chronic sun-damage (high-CSD) and acral and mucosal melanomas often do not arise from a preexisting nevus

needed to determine reasonable genetic and histopathologic definitions for atypical Spitz tumors (Spitz melanocytomas) and their associated biologic potential.

\section{Melanocytomas in common congenital nevi}

A number of secondary growths can develop within common congenital nevi. Neuroid overgrowths are slowly growing soft nodules that develop slowly over years [93]. They typically blend in with the surrounding common congenital nevus, and it is not known whether they harbor additional genetic alterations. Proliferative nodules grow rapidly and may ulcerate, clinically mimicking melanomas. In some cases, they consist of hypercellular nodules of melanocytes, often with enlarged and hyperchromatic nuclei and occasionally with numerous mitotic figures and necrosis. Despite the presence of alarming clinical and histopathologic features, proliferative nodules follow a benign course in contrast to melanomas that arise within congenital nevi that have a poor prognosis. Proliferative nodules often demonstrate losses and gains of entire chromosomes, in contrast to common congenital nevi that typically do not demonstrate copy number alterations $[93,94]$. This finding indicates that proliferative nodules are subclonal outgrowths with additional genetic alterations. Copy number analysis can be helpful for distinguishing proliferative nodules from melanoma because losses and gains of subchromosomal regions are characteristic of melanoma but not proliferative nodules [94]. Specific driver mutations beyond those found in nevi have not been identified in proliferative nodules. Proliferative nodules do not display the distinctive histopathologic features of deep penetrating or $B A P 1$-inactivated melanocytoma, both 
of which have been reported to arise within congenital nevi $[95,96]$.

\section{Discussion}

In this review we have discussed the clinical, histopathologic and genetic features of various subtypes of acquired and congenital melanocytic nevi, as well as the new genetically defined concept of melanocytoma. Acquired nevi arise by the acquisition of a single oncogenic mutation within a melanocyte or melanocyte precursor after birth; their congenital counterparts result from acquisition of the same oncogenic mutation in a melanocyte or melanocyte precursor during development. All the initiating nevusassociated mutations we have discussed here activate the MAPK pathway, highlighting the central importance of this pathway in melanocytic neoplasia.

In our new paradigm of tumor classification, there are multiple pathways of genetic progression to melanoma (Fig. 6). The common acquired nevus is part of the lowchronic sun damage pathway with UV radiation exposure contributing the nevus formation. The melanocytomas that arise within this pathway harbor secondary mutations that activate protein kinase A (PRKARIA- inactivated melanocytoma) or the beta-catenin pathway (deep penetrating or betacatenin-activated melanocytoma) or perturb the epigenetic state of the genome (BAPl-inactivated melanocytoma). The presence of a common nevus adjacent to one of these melanocytomas indicates that the nevus-initiating mutation occurred first with one of the melanocytes of the common nevus later acquiring additional mutation(s) which drove a second wave of subclonal expansion. Melanocytomas do occur in the absence of a common nevus suggesting that the non-MAPK pathway-activating mutation(s) may sometimes occur first. It is possible that these non-MAPK pathway-activating mutations do not result in a clinically detectable melanocytic tumor, but when the mutated melanocyte subsequently acquires a tumor-initiating MAPK pathway activating mutation, a clinically detectable tumor develops. Melanocytomas can also develop from nevi in other pathways of melanoma development, including Spitz nevi and congenital nevi.

Our clinical experience with the melanocytomas discussed in this review is that they have benign or low-grade biologic behavior. Based on their genetics, we expect that they have an increased risk of malignant transformation as compared to their nevus counterparts but their absolute risk of transformation is not established and it is unclear if it is high enough to justify routine re-excision to prevent persistence. The secondary alterations in these melanocytomas span only a small fraction of the known oncogenic mutations that occur in cutaneous melanoma. Additional genetic study of diagnostically challenging melanocytic tumors, such as dysplastic nevi, may reveal additional categories of melanocytomas, perhaps even those with activation or disruption of more than two biologic pathways. The biologic potential of various categories of melanocytomas needs to be determined by careful clinical observational studies in order to guide optimal patient management.

Acknowledgements I am very grateful to my many generous colleagues and mentors over the years. My work with Dr. Boris Bastian has greatly informed my view of the genetics of melanocytic tumors. Dr. Philip LeBoit provided micrographs. Thank you to Dr. Richard Jordan for careful reading of the manuscript and helpful suggestions. This work was supported by the National Cancer Institute at the National Institutes of Health (grant number 1R35CA220481).

\section{Compliance with ethical standards}

Conflict of interest The author declares that she has no conflict of interest.

Publisher's note Springer Nature remains neutral with regard to jurisdictional claims in published maps and institutional affiliations.

\section{References}

1. Pollock PM, Harper UL, Hansen KS, et al. High frequency of BRAF mutations in nevi. Nat Genet. 2003;33:19-20.

2. Van Raamsdonk CD, Bezrookove V, Green G, et al. Frequent somatic mutations of GNAQ in uveal melanoma and blue naevi. Nature. 2009;457:599-602.

3. Yeh I, von Deimling A, Bastian BC. Clonal BRAF mutations in melanocytic nevi and initiating role of BRAF in melanocytic neoplasia. J Natl Cancer Inst. 2013;105:917-9.

4. Van Raamsdonk CD, Griewank KG, Crosby MB, et al. Mutations in GNA11 in uveal melanoma. N Engl J Med. 2010;363:2191-9.

5. Wiesner T, He J, Yelensky R, et al. Kinase fusions are frequent in Spitz tumours and spitzoid melanomas. Nat Commun. 2014;5:3116.

6. Hanahan D, Weinberg RA. Hallmarks of cancer: the next generation. Cell. 2011;144:646-74.

7. Bataille V, Kato BS, Falchi M, et al. Nevus size and number are associated with telomere length and represent potential markers of a decreased senescence in vivo. Cancer Epidemiol Biomark Prev. 2007;16:1499-502.

8. Duffy DL, Zhu G, Li X, et al. Novel pleiotropic risk loci for melanoma and nevus density implicate multiple biological pathways. Nat Commun. 2018;9:4774.

9. Wachsmuth RC, Turner F, Barrett JH, et al. The effect of sun exposure in determining nevus density in UK adolescent twins. $\mathrm{J}$ Invest Dermatol. 2005;124:56-62.

10. Newton-Bishop JA, Chang Y-M, Iles MM, et al. Melanocytic nevi, nevus genes, and melanoma risk in a large case-control study in the United Kingdom. Cancer Epidemiol Biomark Prev. 2010;19:2043-54.

11. Li W-Q, Cho E, Weinstock MA, et al. Cutaneous nevi and risk of melanoma death in women and men: a prospective study. J Am Acad Dermatol. 2019;80:1284-91.

12. Massi G, LeBoit PE. Histological diagnosis of nevi and melanoma. Berlin, Heidelberg: Springer; 2014.

13. Rongioletti F, Urso C, Batolo D, et al. Melanocytic nevi of the breast: a histologic case-control study. J Cutan Pathol. 2004;31: $137-40$. 
14. Rongioletti F, Ball RA, Marcus R, et al. Histopathological features of flexural melanocytic nevi: a study of 40 cases. J Cutan Pathol. 2000;27:215-7.

15. Fabrizi G, Pagliarello C, Parente P, et al. Atypical nevi of the scalp in adolescents. J Cutan Pathol. 2007;34:365-9.

16. Clark WH Jr., Hood AF, Tucker MA, et al. Atypical melanocytic nevi of the genital type with a discussion of reciprocal parenchymal-stromal interactions in the biology of neoplasia. Hum Pathol. 1998;29:S1-24.

17. Kerl H, Trau H, Ackerman AB. Differentiation of melanocytic nevi from malignant melanomas in palms, soles, and nail beds solely by signs in the cornified layer of the epidermis. Am J Dermatopathol. 1984;6(Suppl:):159-60.

18. Boyd AS, Rapini RP. Acral melanocytic neoplasms: a histologic analysis of 158 lesions. J Am Acad Dermatol. 1994;31:740-5.

19. Requena C, Requena L, Kutzner H, et al. Spitz nevus: a clinicopathological study of 349 cases. Am J Dermatopathol 2009; 31:107-16.

20. Herreid PA, Shapiro PE. Age distribution of spitz nevus vs. malignant melanoma. Arch Dermatol. 1996;132:352-3.

21. Paniago-Pereira C, Maize JC, Ackerman AB. Nevus of large spindle and/or epithelioid cells (Spitz's nevus). Arch Dermatol. 1978;114:1811-23.

22. Weedon D, Little JH. Spindle and epithelioid cell nevi in children and adults. A review of 211 cases of the spitz nevus. Cancer. 1977;40:217-25.

23. Spitz S. Melanomas of childhood. Am J Pathol. 1948;24:591-609.

24. Bastian BC, LeBoit PE, Pinkel D. Mutations and copy number increase of HRAS in Spitz nevi with distinctive histopathological features. Am J Pathol. 2000;157:967-72.

25. Botton T, Yeh I, Nelson T, et al. Recurrent BRAF kinase fusions in melanocytic tumors offer an opportunity for targeted therapy. Pigment Cell Melanoma Res. 2013;26:845-51.

26. Yeh I, Botton T, Talevich E, et al. Activating MET kinase rearrangements in melanoma and Spitz tumours. Nat Commun. 2015;6:7174

27. Yeh I, Tee MK, Botton T, et al. NTRK3 kinase fusions in Spitz tumours. J Pathol. 2016;240:282-90.

28. VandenBoom T, Quan VL, Zhang B, et al. Genomic fusions in pigmented spindle cell nevus of reed. Am J Surg Pathol 2018;42:1042-51.

29. Quan VL, Zhang B, Mohan LS, et al. Activating structural alterations in MAPK genes are distinct genetic drivers in a unique subgroup of spitzoid neoplasms. Am J Surg Pathol 2019. https:// doi.org/10.1097/PAS.0000000000001213.

30. Newman S, Fan L, Pribnow A, et al. Clinical genome sequencing uncovers potentially targetable truncations and fusions of MAP3K8 in spitzoid and other melanomas. Nat Med 2019; 25:597-602.

31. Busam KJ, Kutzner H, Cerroni L, et al. Clinical and pathologic findings of Spitz nevi and atypical Spitz tumors with ALK fusions. Am J Surg Pathol. 2014;38:925-33.

32. Yeh I, de la Fouchardiere A, Pissaloux D, et al. Clinical, histopathologic, and genomic features of Spitz tumors with ALK fusions. Am J Surg Pathol. 2015;39:581-91.

33. Amin SM, Haugh AM, Lee CY, et al. A comparison of morphologic and molecular features of BRAF, ALK, and NTRK1 fusion spitzoid neoplasms. Am J Surg Pathol. 2017; 41:491-8.

34. Yeh I, Busam KJ, McCalmont TH, et al. Filigree-like rete ridges, lobulated nests, rosette-like structures, and exaggerated maturation characterize spitz tumors with NTRK1 fusion. Am J Surg Pathol. 2019;43:737-46.

35. Möller I, Murali R, Müller H, et al. Activating cysteinyl leukotriene receptor 2 (CYSLTR2) mutations in blue nevi. Mod Pathol 2016. https://doi.org/10.1038/modpathol.2016.201.
36. Griewank KG, Müller H, Jackett LA, et al. $S F 3 B 1$ and $B A P 1$ mutations in blue nevus-like melanoma. Mod Pathol. 2017;30:928.

37. Bastian BC. The molecular pathology of melanoma: an integrated taxonomy of melanocytic neoplasia. Annu Rev Pathol. 2014;9: 239-71.

38. Baykal C, Yilmaz Z, Sun GP, et al. The spectrum of benign dermal dendritic melanocytic proliferations. J Eur Acad Dermatol Venereol. 2019;33:1029-41.

39. Karvonen SL, Vaajalahti P, Marenk M, et al. Birthmarks in 4346 Finnish newborns. Acta Derm Venereol. 1992;72:55-7.

40. Castilla EE, da Graça Dutra M, Orioli-Parreiras IM. Epidemiology of congenital pigmented naevi: II. Risk factors. Br J Dermatol. 1981;104:421-7.

41. Krengel S, Scope A, Dusza SW, et al. New recommendations for the categorization of cutaneous features of congenital melanocytic nevi. J Am Acad Dermatol. 2013;68:441-51.

42. Bauer J, Curtin JA, Pinkel D, et al. Congenital melanocytic nevi frequently harbor NRAS mutations but no BRAF mutations. J Invest Dermatol. 2007;127:179-82.

43. Salgado CM, Basu D, Nikiforova M, et al. BRAF mutations are also associated with neurocutaneous melanocytosis and large/giant congenital melanocytic nevi. Pediatr Dev Pathol. 2015;18:1-9.

44. Sarin KY, Sun BK, Bangs CD, et al. Activating hras mutation in agminated spitz nevi arising in a nevus spilus. JAMA Dermatol. 2013;149:1077-81.

45. Dessars B, De Raeve LE, El Housni H, et al. Chromosomal translocations as a mechanism of BRAF activation in two cases of large congenital melanocytic nevi. J Invest Dermatol. 2007;127: $1468-70$.

46. Martins da Silva V, Martinez-Barrios E, Tell-Martí G, et al. Genetic abnormalities in large to giant congenital nevi: beyond NRAS mutations. J Investig Dermatol 2018. https://doi.org/10. 1016/j.jid.2018.07.045.

47. Baltres A, Salhi A, Houlier A, et al. Malignant melanoma with areas of rhabdomyosarcomatous differentiation arising in a giant congenital nevus with RAF1 gene fusion. Pigment Cell Melanoma Res 2019. https://doi.org/10.1111/pcmr.12785.

48. Radentz WH, Vogel P. Congenital common blue nevus. Arch Dermatol. 1990;126:124-5.

49. Kawasaki T, Tsuboi R, Ueki R, et al. Congenital giant common blue nevus. J Am Acad Dermatol. 1993;28:653-4.

50. Busam KJ, Lohmann CM. Congenital pauci-melanotic cellular blue nevus. J Cutan Pathol. 2004;31:312-7.

51. Lee MY, Jin S, Lee K-H, et al. A cellular blue nevus with pigmented epithelioid melanocytoma-like pattern on the ipsilateral upper arm associated with a congenital plaque-type blue nevus on the hand. J Cutan Pathol. 2019;46:383-8.

52. Findler G, Hoffman HJ, Thomson HG, et al. Giant nevus of the scalp associated with intracranial pigmentation. Case report. J Neurosurg. 1981;54:108-12.

53. Marano SR, Brooks RA, Spetzler RF, et al. Giant congenital cellular blue nevus of the scalp of a newborn with an underlying skull defect and invasion of the dura mater. Neurosurgery. 1986;18:85-9.

54. Madaree A, Ramdial PK, Du Trevou M. Giant congenital naevus of the scalp and cranium: case report and review of the literature. Br J Plast Surg. 1997;50:20-5.

55. Micali G, Innocenzi D, Nasca MR. Cellular blue nevus of the scalp infiltrating the underlying bone: case report and review. Pediatr Dermatol. 1997;14:199-203.

56. Kurokawa R, Kim P, Kawamoto $\mathrm{T}$, et al. Intramedullary and retroperitoneal melanocytic tumor associated with congenital blue nevus and nevus flammeus: an uncommon combination of neurocutaneous melanosis and phacomatosis pigmentovascularis-case report. Neurol Med Chir. 2013;53:730-4. 
57. Küsters-Vandevelde HVN, van Engen-van Grunsven IACH, Coupland SE, et al. Mutations in g protein encoding genes and chromosomal alterations in primary leptomeningeal melanocytic neoplasms. Pathol Oncol Res. 2015;21:439-47.

58. Thomas AC, Zeng Z, Rivière J-B, et al. Mosaic activating mutations in GNA11 and GNAQ are associated with phakomatosis pigmentovascularis and extensive dermal melanocytosis. J Investig Dermatol. 2016;136:770-8.

59. Kumar A, Zastrow DB, Kravets EJ, et al. Extracutaneous manifestations in phacomatosis cesioflammea and cesiomarmorata: case series and literature review. Am J Med Genet Part A. 2019;179:966-77.

60. Shirley MD, Tang H, Gallione CJ, et al. Sturge-Weber syndrome and port-wine stains caused by somatic mutation in GNAQ. N Engl J Med. 2013;368:1971-9.

61. Groesser L, Peterhof E, Evert M, et al. BRAF and RAS mutations in sporadic and secondary pyogenic granuloma. J Invest Dermatol. 2016;136:481-6.

62. Liau J-Y, Lee J-C, Tsai J-H, et al. High frequency of GNA14, GNAQ, and GNA11 mutations in cherry hemangioma: a histopathological and molecular study of 85 cases indicating GNA14 as the most commonly mutated gene in vascular neoplasms. Mod Pathol 2019. https://doi.org/10.1038/s41379-019-0284-y.

63. Elder DE, Massi D, Scolyer R, et al. WHO classification of skin tumours. 4th ed. Lyon, France: IARC Press; 2018.

64. Zembowicz A, Carney JA, Mihm MC. Pigmented epithelioid melanocytoma: a low-grade melanocytic tumor with metastatic potential indistinguishable from animal-type melanoma and epithelioid blue nevus. Am J Surg Pathol. 2004;28:31-40.

65. Zembowicz A, Knoepp SM, Bei T, et al. Loss of expression of protein kinase a regulatory subunit 1alpha in pigmented epithelioid melanocytoma but not in melanoma or other melanocytic lesions. Am J Surg Pathol. 2007;31:1764-75.

66. Mandal RV, Murali R, Lundquist KF, et al. Pigmented epithelioid melanocytoma: favorable outcome after 5-year follow-up. Am J Surg Pathol. 2009;33:1778-82.

67. Cohen JN, Joseph NM, North JP, et al. Genomic analysis of pigmented epithelioid melanocytomas reveals recurrent alterations in PRKAR1A, and PRKCA genes. Am J Surg Pathol. 2017; 41:1333-46.

68. Isales MC, Mohan LS, Quan VL, et al. Distinct genomic patterns in pigmented epithelioid melanocytoma: a molecular and histologic analysis of 16 cases. Am J Surg Pathol. 2019;43:480-8.

69. Kirschner LS, Carney JA, Pack SD, et al. Mutations of the gene encoding the protein kinase A type I-alpha regulatory subunit in patients with the Carney complex. Nat Genet. 2000;26:89-92.

70. Seab JA, Graham JH, Helwig EB. Deep penetrating nevus. Am J Surg Pathol. 1989;13:39-44.

71. Yeh I, Lang UE, Durieux E, et al. Combined activation of MAP kinase pathway and $\beta$-catenin signaling cause deep penetrating nevi. Nat Commun. 2017;8:644.

72. High WA, Alanen KW, Golitz LE. Is melanocytic nevus with focal atypical epithelioid components (clonal nevus) a superficial variant of deep penetrating nevus? J Am Acad Dermatol. 2006;55:460-6.

73. Dadras SS, Lu J, Zembowicz A, et al. Histological features and outcome of inverted type-A melanocytic nevi. J Cutan Pathol 2018. https://doi.org/10.1111/cup.13106.

74. Cerroni L, Barnhill R, Elder D, et al. Melanocytic tumors of uncertain malignant potential. Am J Surg Pathol. 2010;34:314-26.

75. Wiesner T, Obenauf AC, Murali R, et al. Germline mutations in BAP1 predispose to melanocytic tumors. Nat Genet. 2011; 43:1018-21.
76. Busam KJ, Sung J, Wiesner T, et al. Combined BRAF(V600E)positive melanocytic lesions with large epithelioid cells lacking BAP1 expression and conventional nevomelanocytes. Am J Surg Pathol. 2013;37:193-9.

77. Yeh I, Mully TW, Wiesner T, et al. Ambiguous melanocytic tumors with loss of 3p21. Am J Surg Pathol. 2014;38:1088-95.

78. Wiesner T, Murali R, Fried I, et al. A distinct subset of atypical spitz tumors is characterized by BRAF mutation and loss of BAP1 expression. Am J Surg Pathol. 2012;36:818-30.

79. Abdel-Rahman MH, Pilarski R, Cebulla CM, et al. Germline BAP1 mutation predisposes to uveal melanoma, lung adenocarcinoma, meningioma, and other cancers. J Med Genet 2011. https://doi.org/10.1136/jmedgenet-2011-100156.

80. Carbone M, Yang H, Pass HI, et al. BAP1 and cancer. Nat Rev Cancer. 2013;13:153-9.

81. Popova T, Hebert L, Jacquemin V, et al. Germline BAP1 mutations predispose to renal cell carcinomas. Am J Hum Genet 2013. https://doi.org/10.1016/j.ajhg.2013.04.012.

82. Harvell JD, Meehan SA, LeBoit PE. Spitz's nevi with halo reaction: a histopathologic study of 17 cases. J Cutan Pathol. 1997;24:611-9.

83. Requena C, Sanz V, Nagore E, et al. BAP1-deficient and VE1-negative atypical Spitz tumor. J Cutan Pathol. 2015;42:564-7.

84. Busam KJ, Murali R, Pulitzer M, et al. Atypical spitzoid melanocytic tumors with positive sentinel lymph nodes in children and teenagers, and comparison with histologically unambiguous and lethal melanomas. Am J Surg Pathol. 2009;33:1386-95.

85. Duncan LM. Atypical Spitz tumours and sentinel lymph nodes. Lancet Oncol. 2014;15:377-8.

86. Fullen DR, Poynter JN, Lowe L, et al. BRAF and NRAS mutations in spitzoid melanocytic lesions. Mod Pathol. 2006;19:1324-32.

87. Da Forno PD, Pringle JH, Fletcher A, et al. BRAF, NRAS and HRAS mutations in spitzoid tumours and their possible pathogenetic significance. Br J Dermatol. 2009;161:364-72.

88. Lazova R, Pornputtapong N, Halaban R, et al. Spitz nevi and Spitzoid melanomas: exome sequencing and comparison with conventional melanocytic nevi and melanomas. Mod Pathol 2017. https://doi.org/10.1038/modpathol.2016.237.

89. Gerami P, Scolyer RA, Xu X, et al. Risk assessment for atypical spitzoid melanocytic neoplasms using FISH to identify chromosomal copy number aberrations. Am J Surg Pathol. 2013;37:676-84.

90. Lee S, Barnhill RL, Dummer R, et al. TERT promoter mutations are predictive of aggressive clinical behavior in patients with spitzoid melanocytic neoplasms. Sci Rep. 2015;5:11200.

91. Gerami P, Cooper C, Bajaj S, et al. Outcomes of atypical spitz tumors with chromosomal copy number aberrations and conventional melanomas in children. Am J Surg Pathol. 2013;37:1387-94.

92. Shain AH, Yeh I, Kovalyshyn I, et al. The genetic evolution of melanoma from precursor lesions. New Engl J Med. 2015; 373:1926-36.

93. Kinsler VA, O'Hare P, Bulstrode N, et al. Melanoma in congenital melanocytic naevi. Br J Dermatol. 2017;176:1131-43.

94. Bastian BC, Xiong J, Frieden IJ, et al. Genetic changes in neoplasms arising in congenital melanocytic nevi: differences between nodular proliferations and melanomas. Am J Pathol. 2002;161:1163-9.

95. Murphy MJ, Jen M, Chang MW, et al. Molecular diagnosis of a benign proliferative nodule developing in a congenital melanocytic nevus in a 3-month-old infant. J Am Acad Dermatol. 2008; 59:518-23.

96. Rocas D, Castillo C, Lamarca S, et al. Unpigmented nodule with loss of BAP1 expression in a medium-sized congenital nevus. Eur J Dermatol. 2015;25:201-2. 